\title{
Unconventional abdominal uses of FIESTA (CISS) sequence
}

\section{Anitha Sen}

Department of Radiodiagnosis, Government Medical College, Kottayam, Kerala, India

Correspondence: Dr. Anitha Sen, Radiodiagnosis, Government Medical College, Kottayam - 686 008, Kerala, India. E-mail: dranithasen@hotmail.com

\begin{abstract}
The ability to provide cross-sectional imaging, combined with a lack of ionizing radiation has made magnetic resonance imaging (MRI) of abdomen popular. We report four interesting cases: Midgut malrotation with volvulus, sigmoid volvulus, biliary rupture of hydatid cyst, and small bowel lipomatosis, where fast imaging employing steady-state acquisition [FIESTA]/constructive interference into steady state [CISS] sequence helped in clinching the diagnosis.
\end{abstract}

Key words: Abdominal MRI; constructive interference into steady state; fast imaging employing steady-state acquisition

\section{Introduction}

Cross-sectional imaging has been increasingly used for the last few years in abdominal evaluation as whole bowel can be seen without overlap and pathology outside lumen can be evaluated. Awareness of risks of ionizing radiation in computed tomography (CT) has prompted exploration of magnetic resonance imaging (MRI); improvements in MR software and hardware have enabled MRI to assume a major role.

MRI provides better soft-tissue contrast compared to CT. Hence, many abnormalities may be detected on MRI, without using intravenous contrast; this may be helpful in patients who are pregnant or have a low glomerular filtration rate (impaired renal function). Multiphase techniques may demonstrate temporal changes in bowel distension. MRI also has a relatively safe intravenous contrast agent profile. Limitations of MRI include cost, imager access, variation in quality of exam (related to patient cooperation and breath-holding ability) and lower spatial

\begin{tabular}{|l|l|}
\hline \multicolumn{2}{|c|}{ Access this article online } \\
\hline Quick Response Code: & \\
\hline & Website: \\
\hline & www.iji.org \\
& DOI: \\
\hline
\end{tabular}

and temporal resolution compared to CT. MRI is indicated in patients with Crohn's disease, polyposis syndromes and other chronic conditions (patients may present at young age, and require multiple serial examinations to assess progression, detect complications and monitor treatment respons $\left.\mathrm{e}^{[1]}\right)$, those for whom exposure to radiation is a concern and those with low-grade small bowel obstruction.

With the increasing referral for MRI abdomen in clinical practice, especially for nonspecific abdominal pain, we are bound to come across complex/interesting cases in MRI. Diagnosis may be missed on MRI unless special care is taken.

Fast gradient echo (GRE) sequences are of mainly two types: Spoiled/incoherent and steady state/coherent. Balanced sequences are a type of steady-state sequence in which gradients applied in three axes are balanced. Due to balancing of gradients in three axes, they are relatively insensitive to motion (like respiration and peristalsis). Examples: Fast imaging employing steady-state acquisition (FIESTA; GE Healthcare, Milwaukee, WI, United States)/fast imaging with steady-state precession (True FISP; Siemens Medical Solutions, Erlangen, Germany)/fast field echo (balanced FFE; Philips Healthcare, Best, the Netherlands) is a basic fully refocused steady-state sequence, and constructive interference into steady state (CISS)/FIESTA-C is a modification. ${ }^{[2]}$ CISS can minimize the dark banding artifacts of steady-state free precession (SSFP) sequences 
that are more prominent on 3T. ${ }^{[3]}$ Initially developed for cardiac imaging, these SSFP sequences are now a cornerstone for neurovascular MRI. They help in differentiating extra-axial from intra-axial cranial lesions and in their characterization; they are also used in imaging of abdomen, fetus, cartilage and breast.

FIESTA has proved to be useful in abdominal imaging for MR small bowel follow-through ${ }^{[4]}$ (duodenal abnormalities including volvulus) and MR enteroclysis, ${ }^{[5-7]}$ MR colonography, oncologic imaging ${ }^{[8]}$ (especially useful in retroperitoneal tumor and pancreatic carcinoma due to its excellent depiction of vascular anatomy) and assessing vascular patency ${ }^{[9]}$ (portal vein). It is compared with single-shot fast spin echo (SSFSE), a commonly used sequence in abdominal MRI, in the following table.

\section{Case 1}

A 38-year-old male presented with vague epigastric pain. MRI (1.5 T) axial FIESTA images [Figure 1A] showed superior mesenteric vein (SMV) swirling around superior mesenteric artery (SMA), which is suggestive of midgut volvulus. This was confirmed by plain CT [Figure 1B-D].

\section{FIESTA}

\section{1) Fast gradient echo}

2) Due to shorter acquisition time, less susceptible to respiratory motion artifacts

3) Higher signal to noise ratio (SNR)

4) Image contrast is $T 1 / T 2$ for longer $T R$ Primarily T2 weighted for short TR and TE In addition to fluid-filled structures seen in SSFSE, solid lesions are also seen well due to better T2 contrast

5) More technically complicated

a) Poor scanner performance can ruin quality (B homogeneity, gradients)

b) Motion is a problem

6) Significantly lower RF absorption (advantage in foetal MRI) ${ }^{[1]}$

7) In foetal MRI, ${ }^{[9]}$

a) Clear differentiation of muscle, bone and cartilage

b) Vascular structures of liver are seen better

c) Both chambers of heart and lungs hyperintense

d) Both small and large bowel hyperintense

8) Cine 2D FIESTA gave additional information regarding visceral peristalsis: differentiated dilated gastrointestinal tract from other intra-abdominal cystic lesions; confirmed the nature and level of gastrointestinal obstruction ${ }^{[10]}$

MRI: Magnetic resonance imaging, FIESTA: Fast imaging employing steady-state acquisition, SSFSE: Single-shot fast spin echo

Fast spin echo

More susceptible

Typically T2 weighted effect is dark
Coronal FIESTA images [Figure 1E and F] showed prominent duodenal loop that returned to right side of the abdomen (suggestive of volvulus); coronal T2 fat-saturated images [Figure 1G and $\mathrm{H}$ ] showed jejunal loops on right and ileal loops on the left of abdomen (suggestive of malrotation).

\section{Discussion}

Malrotation of intestine, seen in approximately 1 in 500 live births, is often asymptomatic. But diagnosing it is important due to its predisposition for volvulus, which often needs surgical intervention. MRI may be able to diagnose malrotation and volvulus without use of intravenous or oral contrast.

Intestinal malrotation results due to defect in normal rotation and fixation of bowel during fetal life. Absent
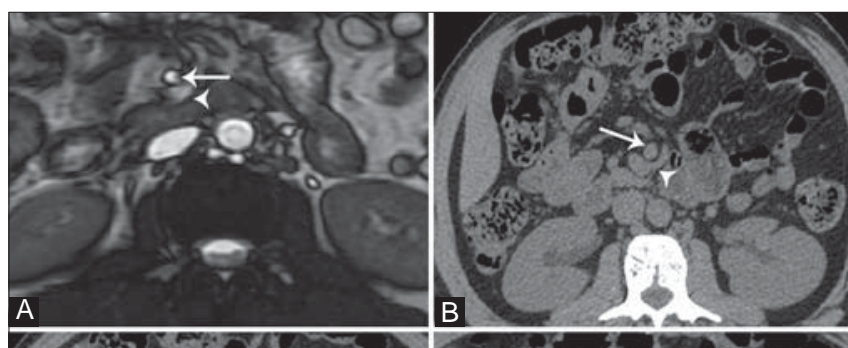

No delineation of bone and soft tissue due to intrinsic blurring

Better demarcation of lungs (for calculation of lung volume)

Differentiation possible: Small bowel is bright and large bowel

Only anatomical information
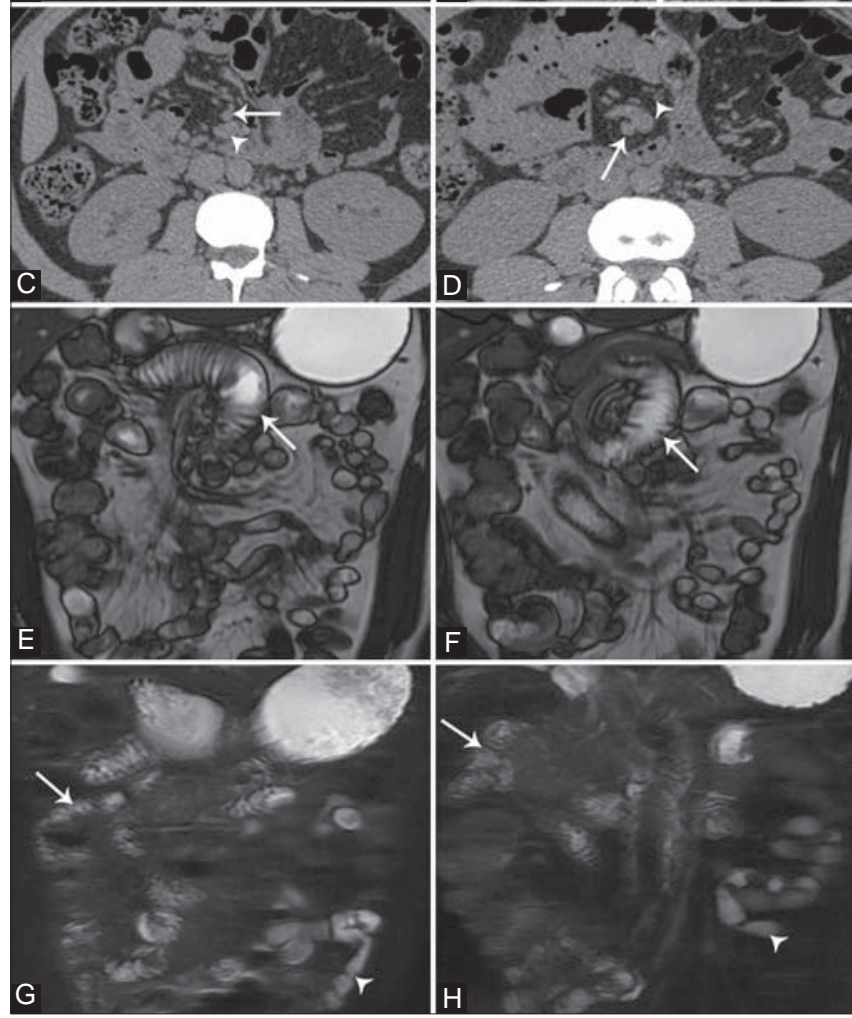

Figure 1 (A-H): A 38-year-old man with malrotation and volvulus. Axia FIESTA image (A) and CT images (B, C, D) show SMV (arrowhead) swirling around SMA (arrow). Coronal FIESTA images (E, F) show prominent duodenal loop (arrow) that returns to the right side of the abdomen. Coronal fat-saturated images $(G, H)$ show jejunal loops on the right (arrow) and ileal loops on the left, (arrowhead) suggestive of malrotation 
or abnormal fixation predisposes bowel and vessels to twisting (volvulus) with consequent vascular compromise and ischemia. This predisposition to volvulus makes diagnosis of malrotation important; close clinical follow-up and timely treatment are indicated.

Diagnosis of bowel malrotation has traditionally been done with barium meal follow-through (BMFT) and contrast-enhanced $\mathrm{CT}$, both of which involve ingestion of oral contrast media. MRI, with its ability to characterize parts of small bowel (differentiate jejunum and ileum), can confidently determine locations of duodenum, jejunum, ileum and large bowel and diagnose malrotation without the use of oral contrast, although bowel distension with polyethylene glycol (PEG) solution given orally (MRI small bowel follow-through ${ }^{[4]}$ ) or through nasojejunal catheter (MR enteroclysis $\left.{ }^{[5-7]}\right)$ is ideal.

Preoperative diagnosis of volvulus is more commonly made by $\mathrm{CT}^{[11-13]}$ than by ultrasound (USG) or MRI, ${ }^{[4,14]}$ by
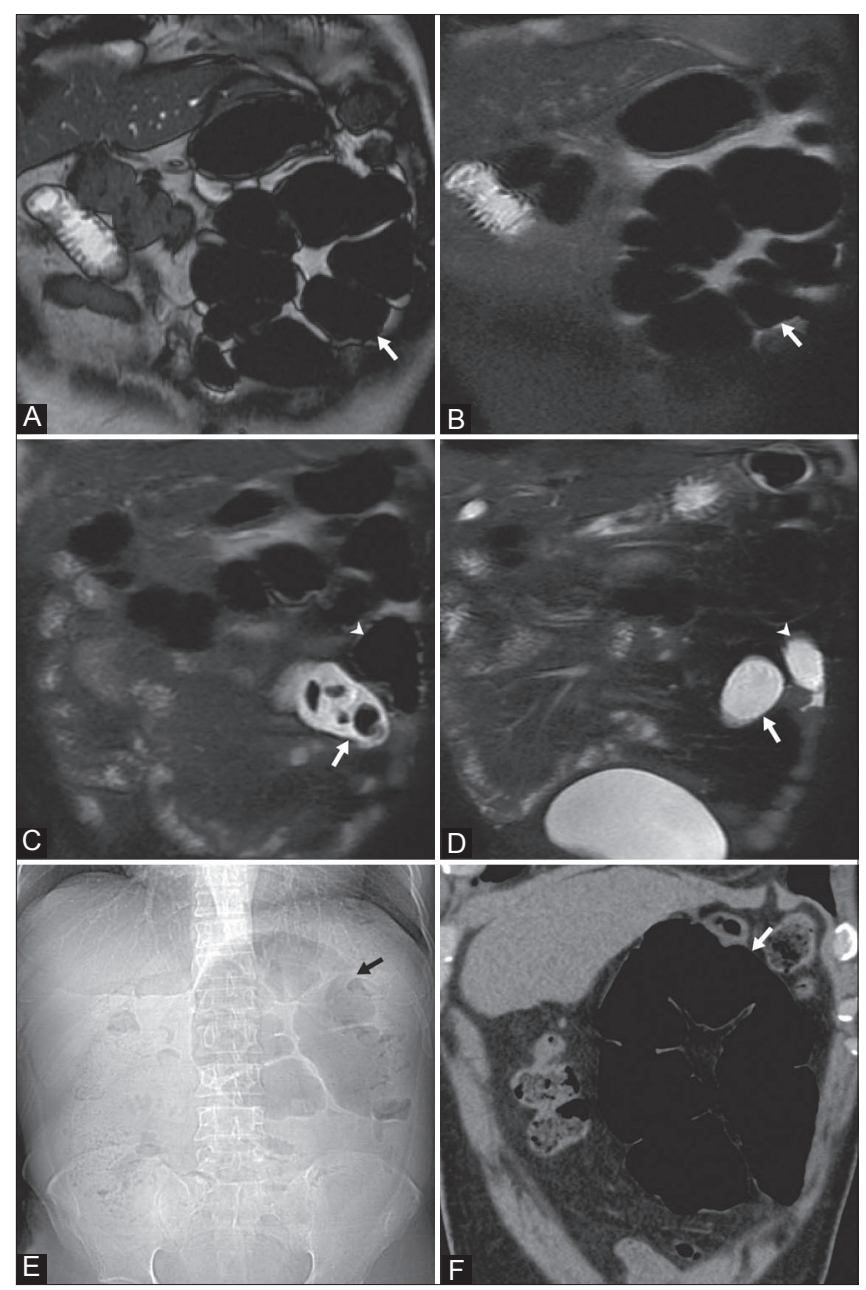

Figure 2 (A-F): A 70-year-old man with sigmoid volvulus. Coronal FIESTA image (A) shows a dilated bowel loop (arrow) in left lower abdomen. Coronal fat-saturated images (B, C, D) after water enema show fluid in limbs (arrow in C, arrow and arrowhead in D) of loop. CT scanogram (E) and coronal CT (F) also confirm dilated loop demonstrating the swirl sign or reversal of SMA-SMV relationship, ${ }^{[12,13]}$ corkscrewing or beaking of bowel. Unlike CT, here patency of SMA and SMV could be demonstrated on MRI (FIESTA) without intravenous contrast or ionizing radiation.

\section{Case 2}

A 70-year-old man presented with abdominal discomfort and history of blood in stools. Coronal MR (1.5 T) FIESTA image [Figure 2A] showed an abnormally dilated bowel loop in left lower abdomen, suggestive of sigmoid volvulus. This was confirmed by coronal T2 fat-saturated images [Figure 2B-D], which showed air-filled loop [Figure 2B] and fluid in the limbs of loop [Figure 2C and D], CT scanogram [Figure 2E], and coronal CT section [Figure 2F].

\section{Discussion}

Sigmoid volvulus, a common cause of large bowel obstruction, generally occurs in adult men (mean age 56-77 years) and is more common in eastern countries (Middle East is called the volvulus belt). Abdominal

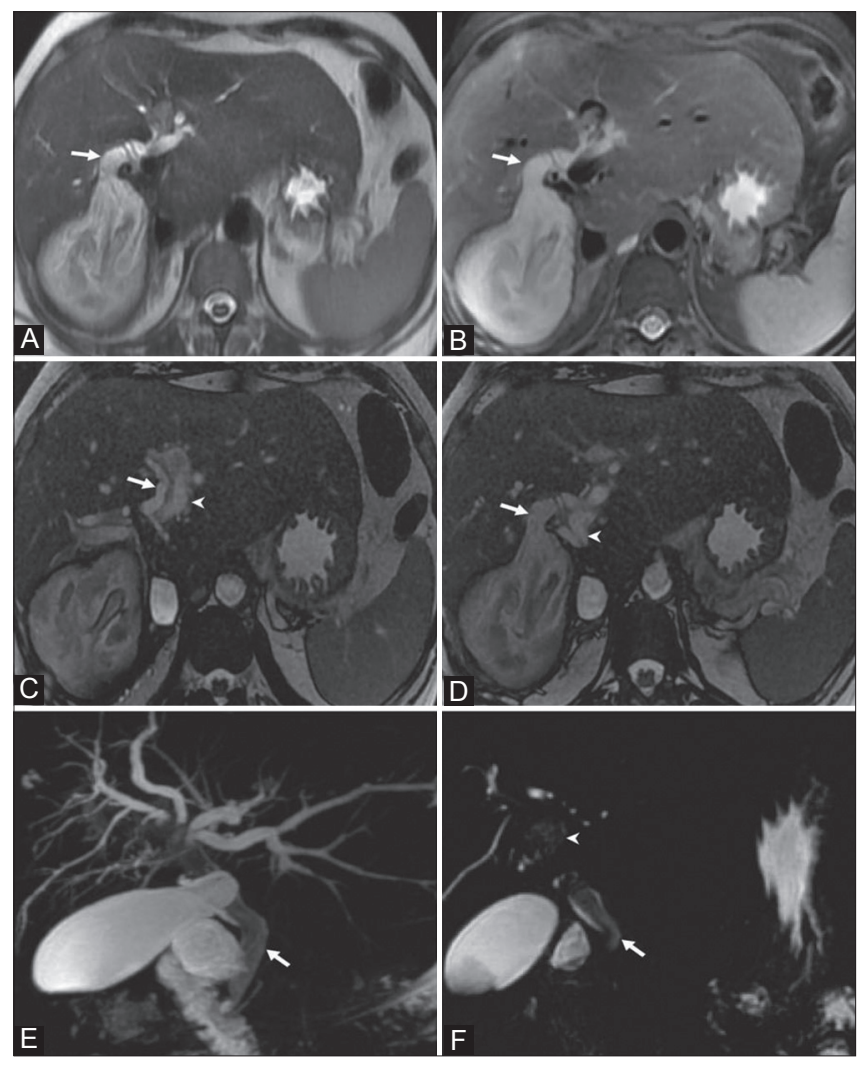

Figure 3 (A-F): A 40-year-old female with biliary rupture of hydatid. Axial T2 (A) and T2 fat-saturated (B) images show hydatid cyst in the right lobe of the liver, extending up to portal hilum with some communication (arrow). Axial FIESTA (C, D) images show that the communication is with biliary tree (arrow); portal venous branches (arrowhead) are seen separately. MRCP images (E, F) show contents (arrow) within CBD, confirming biliary rupture 
radiographs usually show a dilated sigmoid colon and/or multiple small bowel air-fluid levels..$^{[15,16]}$

Since many elderly patients ${ }^{[17]}$ are generally of poor health, with different food and defecation habits, clinical features may be nonspecific, and imaging studies, especially CT and MRI, may help in accurate diagnosis. Non-operative detorsion with flexible endoscopy is the first treatment option, especially in elderly patients; emergency surgery is essential if non-operative treatment is unsuccessful or if peritonitis, bowel gangrene, or perforation is present. Timely detection is aided by FIESTA.

\section{Case 3}

A 40-year-old female presented with acute exacerbation of right upper quadrant pain. MR (3 T) axial T2 [Figure 3A] and T2 fat-saturated [Figure 3B] images showed a hydatid cyst in the right lobe of the liver, reaching up to liver hilum. MR axial FIESTA [Figure 3C and D] images differentiated biliary tract from portal vein branches and clearly demonstrated biliary communication [Figure 3D]. Hydatid material seen within common bile duct (CBD) on magnetic resonance choangiopancreatography (MRCP) images [Figure 3E and F] confirmed biliary rupture.

\section{Discussion}

Hydatid disease caused by larval stage of Echinococcus tapeworm is a major endemic problem in many parts of the world, mainly in sheep-rearing regions. It involves the liver in $75 \%$ cases; right lobe is more frequently $(80 \%)$ involved than the left lobe. Hydatid cyst rupture is a known complication; three types of rupture - contained, communicating and direct - have been described. ${ }^{[18]}$ Frank biliary rupture occurs in 3-17\%; biliary obstruction and cholangitis leads to jaundice, fever and chills.

Communicating biliary rupture may occur into the right duct (55-60\% cases), left duct (25-30\% cases) and rarely into confluence or gall bladder. ${ }^{[19,20]}$ It may be in the form of small fissures/fistulas between biliary radicles and cyst or a wide perforation that allows drainage to a main biliary branch. The only direct sign of cyst rupture is breach in low-signal intensity cyst wall and communication with a biliary radicle. In some cases, hydatid material can be seen passing through the defect and filling the biliary tree. Fluid levels and presence of air in cysts are indirect signs of biliary rupture. When the cyst empties, it may become smaller and less spherical. ${ }^{[18]}$ In case of rupture into gall bladder, presence of collapsed folded membranes in the gall bladder may mimic a soft-tissue mass. ${ }^{[21]}$ Dilated biliary tree is not always due to cyst rupture; it may be due to biliary compression by cyst or associated common bile duct stone. The demonstration of biliary communication is thus very helpful in solving many diagnostic dilemmas.
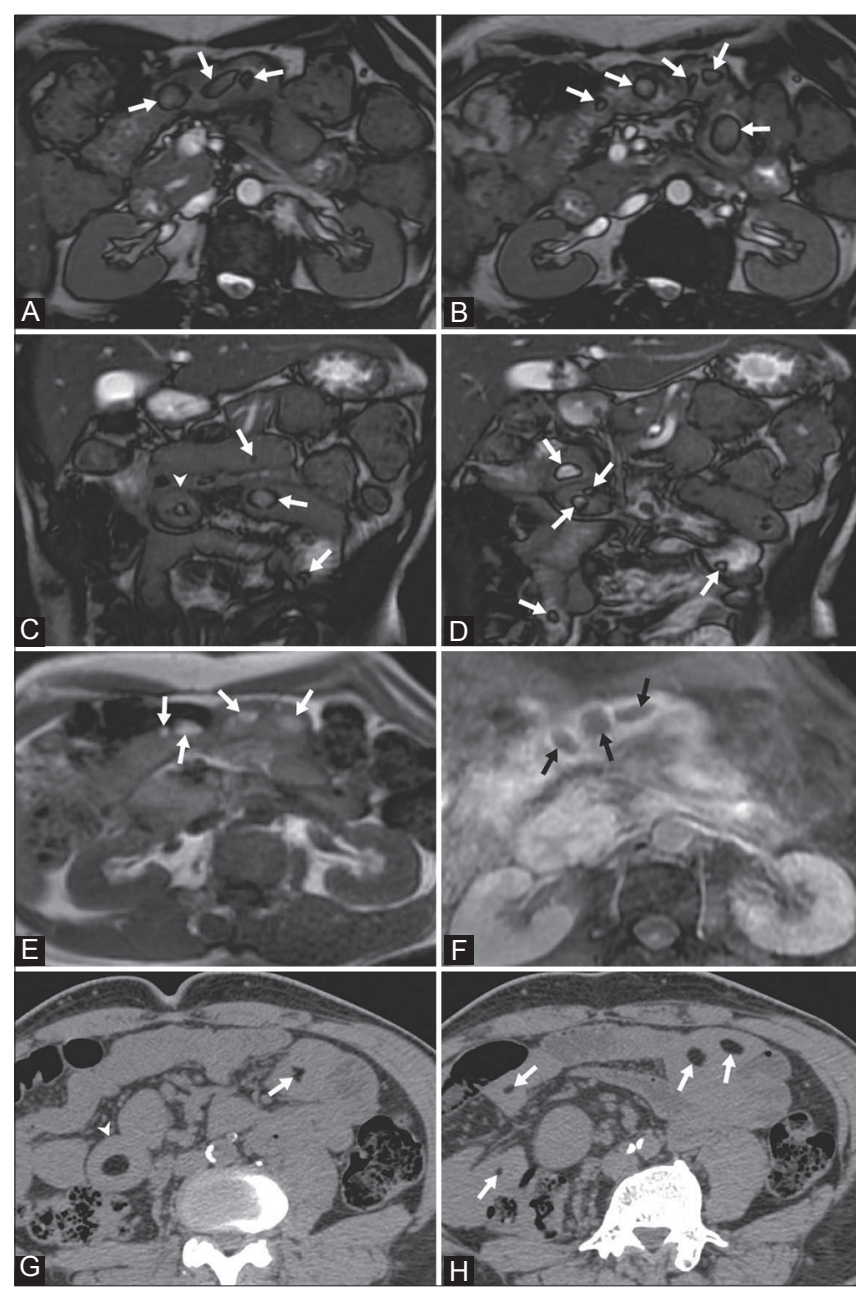

Figure 4(A-H): A 63-year-old male with multiple intestinal lipomas. Axial (A, B) and coronal $(C, D)$ FIESTA images show multiple well-defined areas with dark rim (arrows). Axial T1 image (e) shows hyperintense areas (arrows) which suppress (arrows) on T2 fat-saturated image (f). CT (G, H) confirms fat density of the lesions (arrows). Thickened bowel loop (arrowhead) in (c) and (g) indicate intussusception with lipoma in the center

\section{Case 4}

A 63-year-old male came for follow-up of resolving liver lesion. MR (1.5 T) axial [Figure 4A and B] and coronal FIESTA [Figure 4C and D] images showed well-defined lesions with black outline within small bowel. These lesions were hyperintense on $\mathrm{T} 1$ [Figure $4 \mathrm{E}$ ] and suppressed on fat-saturated images [Figure $4 \mathrm{~F}$ ] suggestive of fat content. CT sections [Figure $4 \mathrm{G}$ and $\mathrm{H}$ ] confirmed the fat density of small bowel lipomas. Wall thickening noted around a lipoma on MR [Figure 4C] and CT [Figure 4G] images suggested intussusception, a known complication of lipoma.

\section{Discussion}

Primary lipomas of small bowel are rare. ${ }^{[22-26]}$ They tend to occur in the elderly ${ }^{[27]}$ (mainly from sixth to seventh decade). Patients may be asymptomatic or present with paroxysmal abdominal pain or gastrointestinal hemorrhage due to complications like intussusception and volvulus. 
Abdominal radiographs may occasionally show intestinal obstruction or low density of lipid. Double-contrast barium studies (like small bowel enema) may show well-defined filling defect within bowel lumen; the shape of mass may change with peristalsis or manual pressure. ${ }^{[24]}$ "Bull's-eye sign" is a niche formation in the radiolucent filling defect due to ulceration. USG may detect intussusception, but it is difficult to diagnose lipomas. ${ }^{[27]} \mathrm{CT}$ showing fat attenuation ${ }^{[27,28]}$ is the definitive investigation. On MRI, intestinal lipomas have been known to be hyperintense on $\mathrm{T} 1-[29,30]$ and $\mathrm{T}^{[30]}$-weighted images and show signal drop-off on fat-suppressed sequences. ${ }^{[4]}$ To the best of our knowledge, FIESTA findings of intestinal lipomas have not been described.

Intracranial lipomas are known to show high signal on T1- and T2-weighted images, suppress on fat-saturated sequences, and show low signal margin on FIESTA sequence due to chemical shift artifact at fat-water interface. We have found similar low signal around intestinal lipomas on FIESTA and they are very useful in drawing attention to the presence of lipomas.

\section{Conclusion}

FIESTA sequence is very useful in abdominal imaging as demonstrated by these interesting cases. Making it a part of routine abdominal MRI protocol is sure to add clarity in equivocal cases.

\section{References}

1. Sempere GA, Martinez Sanjuan V, Medina Chulia E, Benages A, Tome Toyosato A, Canelles P, et al. MRI evaluation of inflammatory activity in Crohn's disease. AJR Am J Roentgenol 2005;184:1829-35.

2. Chavhan GB, Babyn PS, Jankharia BG, Cheng HL, Shroff MM. Steady-state MR imaging sequences: Physics, classification, and clinical applications. Radiographics 2008;28:1147-60.

3. Erturk SM, Alberich-Bayarri A, Herrmann KA, Marti-Bonmati L, Ros PR. Use of 3.0-T MR imaging for evaluation of the abdomen. Radiographics 2009;29:1547-63.

4. Cronin CG, Lohan DG, DeLappe E, Roche C, Murphy JM. Duodenal Abnormalities at MR Small-Bowel Follow-Through. AJR Am J Roentgenol 2008;191:1082-92.

5. Martin DR, Danrad R, Herrmann K, Semelka RC, Hussain SM. Magnetic resonance imaging of the gastrointestinal tract. Top Magn Reson Imaging 2005;16:77-98.

6. Masselli G, Casciani E, Polettini E, Lanciotti S, Bertini L, Gualdi G. Assessment of Crohn's disease in the small bowel: Prospective comparison of magnetic resonance enteroclysis with conventional enteroclysis. Eur Radiol 2006;16:2817-27.

7. Wiarda BM, Kuipers EJ, Heitbrink MA, van Oijen A, Stoker J. MR enteroclysis of inflammatory small-bowel diseases. AJR Am J Roentgenol 2006;187:522-31.

8. Bhosale P, Ma J, Choi H. Utility of the FIESTA Pulse Sequence in Body Oncologic Imaging: Review. AJR Am J Roentgenol 2009;192 (6 Suppl):S83-93.

9. Smith CS, Sheehy N, McEniff N, Keogan MT. Magnetic resonance portal venography: Use of fast-acquisition true FISP imaging in the detection of portal vein thrombosis. Clin Radiol 2007;62:1180-8.

10. Shinmoto H, Tanami Y, Oshio K, Tanimoto A, Higuchi N, Okuda S, et al. Fetal MRI: SSFSE versus FIESTA. Proc Intl Soc Mag Reson Med 2004;11:931.

11. Shen SH, Guo WY, Hung JH. Two-dimensional fast imaging employing steady-state acquisition (FIESTA) cine acquisition of fetal non-central nervous system abnormalities. J Magn Reson Imaging 2007;26:672-7.

12. Jayaraman MV, Mayo-Smith WW, Movson JS, Dupuy DE, Wallach MT. CT of the duodenum: An overlooked segment gets its due. Radiographics 2001;21:S147-60.

13. Nichols DM, Li DK. Superior mesenteric vein rotation: A CT sign of midgut malrotation. AJR Am J Roentgenol 1983;141:707-8.

14. Kawasaki C, Minami A, Yasuda K. A Case of Small Bowel Volvulus Detected by Diagnostic Imaging, which was Useful. J Japan Surg Assoc 1999;60:1023-8.

15. Lal SK, Morgenstern R, Vinjirayer EP, Matin A. Sigmoid volvulus an update. Gastrointest Endoscopy Clin North Am 2006;16:175-87.

16. Heis HA, Bani-Hani KE, Rabadi DK, Elheis MA, Bani-Hani BK, Mazahreh TS, et al. Sigmoid volvulus in the Middle East. World J Surg 2008;32:459-64.

17. Atamanalp SS, Ozturk G. Sigmoid Volvulus in the Elderly: Outcomes of a 43-Year, 453-Patient Experience. Surg Today 2011;41:514-9.

18. Lewall DB, McCorkell SJ. Rupture of echinococcal cysts: Diagnosis, classification and clinical implications. AJR Am J Roentgenol 1986;146:391-4

19. Stavorovsky M, Wientroub S, Iellin A, Papo J. Rupture of hydatid cysts of the liver into the biliary tracts. Int Surg 1977;62:603-8.

20. Becker K, Frieling T, Salch A, Haussinger D. Resolution of hydatid liver cyst by spontaneous rupture into the biliary tract. J Hepatol 1997;26:1408-12.

21. Kumar R, Reddy SN, Thulkar S. Intrabiliary rupture of hydatid cyst: Diagnosis with MRI and hepatobiliary isotope study. Br J Radiol 2002;75:271-4.

22. Kiziltaş S, Yorulmaz E, Bilir B, Enç F, Tuncer I. A remarkable intestinal lipoma case. Ulus Travma Acil Cerrahi Derg 2009;15:399-402.

23. Aminian A, Noaparast M, Mirsharifi R, Bodaghabadi M, Mardany O, Ali FA, et al. Ileal intussusception secondary to both lipoma and angiolipoma: A case report. Cases J 2009;2:7099.

24. Karadeniz Cakmak G, Emre AU, Tascilar O, Bektaş S, Uçan BH, Irkorucu $\mathrm{O}$, et al. Lipoma within inverted Meckel's diverticulum as a cause of recurrent partial intestinal obstruction and hemorrhage: A case report and review of literature. World J Gastroenterol 2007;13:1141-3.

25. Du L, Shah TR, Zenilman ME. Image of the month-quiz case. Intussuscepted transverse colonic lipoma. Arch Surg 2007;142:1221.

26. Gore RM, Mehta UK, Berlin JW, Rao V, Newmark GM. Diagnosis and staging of small bowel tumours. Cancer Imaging 2006;6:209-12.

27. Fang SH, Dong DJ, Chen FH, Jin M, Zhong BS. Small intestinal lipomas: Diagnostic value of multi-slice CT enterography. World J Gastroenterol 2010;16:2677-81.

28. Ross GJ, Amilineni V. Case 26: Jejunojejunal intussusception secondary to a lipoma. Radiology 2000;216:727-30.

29. A Mohamed, N Hassan, N Bhat, M Abukhater, M Uddin. Caecal Lipoma, Unusual Cause of Recurrent Appendicitis, Case Report and Literature Review. The Internet Journal of Gastroenterology. 2008; Vol 8:no.1.

30. Chiba T, Suzuki S, Sato M, Tsukahara M, Saito S, Inomata M, et al. A case of a lipoma in the colon complicated by intussusception. Eur J Gastroenterol Hepatol 2002;14:701-2.

Cite this article as: Sen A. Unconventional abdominal uses of FIESTA (CISS) sequence. Indian J Radiol Imaging 2013;23:386-90.

Source of Support: Nil, Conflict of Interest: None declared. 